\title{
Die Relativität des Krankheitsbegriffs am Beispiel der Neuromedizin
}

\author{
Stefan Evers
}

\section{Einleitung}

Die klinischen Neurowissenschaften (d.h. im engeren Sinne die Fachgebiete Neurologie inklusive Neuropädiatrie, Psychiatrie und Neurochirurgie), die sich ganz besonders mit den Grenzen von Krankheit und Gesundheit, von Empfindung und Körperlichkeit befassen, haben zum Krankheitsbegriff zunehmend relativierende Beobachtungen gemacht, die es gerechtfertigt erscheinen lassen, aus der spezifischen Sicht dieser Fächer, im Folgenden kurz Neuromedizin genannt, zu diesem Begriff Stellung zu nehmen. Dabei ist $z u$ berücksichtigen, dass in der westlichen Medizin keine normative Definition des Begriffs Krankheit besteht und somit nur von der konsensuellen Vorstellung über den Begriff Krankheit ausgegangen werden kann. Dies steht im Widerspruch zum dichotomisierenden Charakter dieses Begriffes, der nur eine Einteilung in gesund und krank zulässt. Letzteres ist aber gerade in der Neuromedizin, wie zu zeigen sein wird, nicht immer möglich; die vielfältig konstruierten Grenzen zwischen gesund und krank stellen sich hier oft weniger hart dar als z. B. in manchen operativen Fachgebieten.

Es sollen daher auf verschiedenen Ebenen Anmerkungen gemacht werden, die sich insbesondere mit dieser Relativität der Krankheitszuordnung beschäftigen und paradigmatisch auch für jüngere Forschungsentwicklungen in anderen Gebieten der Medizin stehen. Mit der Relativität ist die Uneindeutigkeit gemeint, mit der ein biologischer und/oder psychischer Zustand oder Prozess als krankhaft oder als gesund bzw. physiologisch charakterisiert werden kann. ${ }^{1}$

1 Aus Platzgründen kann keine ausführliche Diskussion der Originalliteratur erfolgen, jedoch werden Übersichtsarbeiten zur weiterführenden Beschäftigung mit dem Thema angeführt. 


\section{Krankheit als Konstrukt}

Notwendigerweise muss bei der Definition des Krankheitsbegriffs immer zuerst eine Abgrenzung gegenüber dem Begriff der Diagnose erfolgen. Für Patienten sind diese beiden Begriffe in der Regel synonym. Dies bedeutet, dass Patienten die ihnen mitgeteilte Diagnose häufig auch als Krankheitsentität auffassen. Dies wiederum führt zu typischen Missverständnissen in der Patient-Arzt-Beziehung, da für Ärzte implizit verschiedene biologische Konzepte für dieselbe Diagnose (die oft nur ein klinisches Bild beschreibt, ohne es ätiologisch zu begründen) gemeint sein können. Beispielhaft sei hier die Parkinsonsche Krankheit angeführt, die durch ihre eindrückliche Symptomatik von vielen Patienten selbst erkannt und als Entität wahrgenommen wird. Biologisch gesehen können aber verschiedene degenerative Prozesse zu dem Bild der Parkinsonschen Krankheit führen, was durch akademische diagnostische Klassifikationen auch zum Ausdruck gebracht werden kann, von Patienten und Laien aber häufig nicht nachvollzogen wird. Krankheit in diesem Sinne impliziert somit auch immer eine Diagnose, aber umgekehrt muss nicht immer eine Diagnose eine eindeutig definierte Krankheit implizieren. Vielmehr ist die Attribuierung eines biologischen Zustands, der als beschreibende Diagnose mit einem Begriff belegt werden kann, zu einem Krankheitsbegriff von vielen Faktoren abhängig, die nicht zuletzt einer historischen und gesellschaftlichen Bewertung unterliegen.

Die Relativität der Konstruktion des Krankheitsbegriffs kann sogar noch weitergeführt werden. Die Zuordnung eines biologischen oder psychischen Zustands zu einem Krankheitsbegriff kann sogar durch kulturelle Einflüsse ins Gegenteil verkehrt werden. So ist es medizinhistorisch gut belegt, dass in unserem System eindeutig als krank identifizierte biologische Zustände wie $z$. B. die idiopathische Epilepsie in anderen kulturell-historischen Kontexten, trotz aller behindernden Einschränkungen, als gesund oder sogar als Ausdruck eines qualitativ höherwertigen Seelenzustandes angesehen worden sind. ${ }^{2}$ Hier wird bereits der später noch zu erörternde fließende Übergang des Krankheitsbegriffs zum Begriff der funktionellen Behinderung angesprochen (auf die aktuelle Diskussion über den pejorativen Charakter des Begriffs Behinderung soll hier nicht eingegangen werden, hier ist damit nur die weitgehend objektive Funktionseinschränkung gemeint).

Es lässt sich somit als These postulieren, dass es eine Relativität des Krankheitsbegriffs gibt, d.h. derselbe biologische (oder psychische) Zustand kann zu einer (krankheitsdefinierenden) Diagnose führen oder als physiologisch oder sogar als vorteilhaft angesehen werden.

Diese Relativität des Krankheitsbegriffs gilt in besonderem Maße für Erkrankungen aus dem Bereich der Neuromedizin und dort vor allem für die genetischen Syndrome und (oft nicht eindeutig von diesen abgrenzbar) für die chronischen degenerativen Erkrankungen des zentralen Nervensystems. Gründe hierfür sind, dass neuromedizinische Krankheiten häufig das seelische Erleben betreffen, dass diese Krankheiten häufig Mitteilungen der Be-

2 Jilek-Aall (1999), Vanzan/Paladin (1992). 
troffenen im Verlauf der Erkrankung unmöglich machen und somit viele Symptome spekulativ interpretiert werden müssen und dass die Sichtbarmachung zentraler Prozesse des Gehirns mit der modernen funktionellen Bildgebung immer besser gelingt. Es folgt somit als weitere These, dass diese Uneindeutigkeit der Krankheitsbegriffs insbesondere für chronische Erkrankungen des Nervensystems gilt und häufiger ist als gemeinhin angenommen.

Warum ist es nun wichtig, sich über dieses Konstrukt des Krankheitsbegriffs im Klaren zu sein und seine Relativität im praktischen medizinischen Kontext überhaupt zu berücksichtigen?

Ein Bewusstsein über die Relativität des Krankheitsbegriffs ist besonders relevant für

a) die adäquate Aufklärung von Patientinnen und Patienten, damit diese die Möglichkeit haben, auf die Krankheit zu reagieren und sich mit den Konsequenzen inklusive Stigmatisierung auseinander $\mathrm{zu}$ setzen,

b) die gesellschaftliche Normierung eines biologischen Zustands (und damit die Handlungsnotwendigkeiten, die sich dann automatisch aus einer Krankheit heraus ergeben wie z. B. die sozioökonomischen Auswirkungen),

c) die theoretische Weiterentwicklung des Krankheitsbegriffs.

$\mathrm{Zu}$ (a) Eine adäquate Aufklärung von Patienten über ihre Krankheit und über die zugeordnete Diagnose folgt immer der Unterscheidung zwischen beiden Begriffen und dem Versuch, den Patienten deutlich zu machen, dass manche Diagnosen nicht aufgrund eines biologischen oder psychischen Zustands, sondern einer Konstruktion gestellt werden und dass manche biologische Zustände nicht allein krankheitsdefinierend sind. Hieraus ergeben sich direkte Konsequenzen für die Selbstbestimmtheit von Patienten und für die Sichtweise einer Krankheit durch die soziale Umgebung. Explizite Fragen nach der „Schuld“ an einer Krankheit oder der „Prognose“ durch eine Krankheit stellen sich unter Umständen ganz neu, wenn z. B. Pathomechanismen wie eine angeborene genetische Disposition oder ein irreversibler degenerativer Prozess nachgewiesen werden können.

$\mathrm{Zu}$ (b) Wenn ein bestimmter biologischer Zustand gesellschaftlich als krank normiert wird, zieht dies automatisch Handlungen innerhalb der Gesellschaft nach sich. Die hat u. a. direkte sozioökonomische Konsequenzen. Es ist in diesem Kontext sogar möglich, regulierend auf diese sozioökonomischen Konsequenzen über willkürliche Veränderungen in der Krankheitsdefinition Einfluss zu nehmen. Hervorstechendes Beispiel ist die Definition des kognitiven Abbaus mit zunehmendem Alter als Demenz. Hier ergeben sich aus dem fließenden Übergang von dem physiologischen Alterungsprozess hin zu einer krankhaften Demenz, wie er aus biologischer und aus psychosozialer Sicht exakt beschrieben werden kann, gravierende Auswirkungen z. B. auf die Belastung des Krankenkassen- und Rentensystems.

$\mathrm{Zu}$ (c) Die theoretische Diskussion über den Krankheitsbegriff bedarf ebenfalls der ständigen Überprüfung durch die neuen Erkenntnisse der medizinischen Forschung. Die Normierung eines Zustands als krank hat eben auch Aus- 
wirkungen auf den kulturwissenschaftlichen Diskurs. Krankheit als Schuld, Sünde oder Strafe z. B. unterliegt dem klassischen dichotomen Konzept von krank versus gesund. Gegen diese Dichotomie gibt es in der modernen Neuromedizin immer mehr Einwände, die nicht zuletzt auch von der Lebensqualitätsforschung beeinflusst sind, die den Krankheitsbegriff zunehmend von einem definierten biologischen Zustand entfernt und ihn mehr aus dem funktionellen und affektiven Kontext der Betroffenen heraus erklärt.

Im Folgenden sollen die oben skizzierten Probleme der Krankheitsdefinition an Beispielen aus dem Bereich der Neuromedizin genauer ausgeführt werden. Dies soll durch die folgenden Thesen strukturiert werden:

1. Ein biologischer Zustand bzw. Prozess kann sowohl krankheitsbegründend als auch physiologisch vorteilhaft sein.

2. Krankheiten (als pathologische Prozesse) und physiologische Prozesse können ein Kontinuum darstellen.

3. Der Begriff Krankheit beschreibt häufig einen (historisch) definierten und nicht einen physiologisch determinierten Zustand.

4. Die Attribuierung eines biologischen Zustands als Krankheit kann von (manipulierbaren) statistischen Normen abhängen.

\section{Krankheit als Lebensvorteil und Lebensnachteil}

Im medizintheoretischen Diskurs wird Krankheit als ein auf verschiedenen Ebenen (praktisch, theoretisch, nosologisch) beschreibbarer, aber eben doch eindeutiger bzw. einheitlicher Zustand definiert. Wir kommen jedoch immer mehr zu Erkenntnissen, die denselben biologischen Zustand (darunter sind auch biologische Prozesse subsumiert) sowohl als im herkömmlichen Sinne „krank“ als auch im weiteren Sinne als physiologisch bzw. „gesund“ erscheinen lassen. Diese Erkenntnisse sind insbesondere Folge einer genetischen Forschung, die es uns erlaubt, von einer Prädisposition zu sprechen, die sowohl zu krankhaften (d. h. im evolutionären Kontext „Selektions“nachteilen) als auch zu physiologischen (d. h. im evolutionären Kontext „Selektions“vorteilen) biologischen Zuständen führen kann.

Dies soll am Beispiel der Erkrankung Migräne genauer erläutert werden, bei der sicherlich ein Konsens besteht (zumindest bei den von Migräneattacken Betroffenen), dass es sich bei einer Attacke selbst um einen krankhaften Zustand handelt.

Die Migräne ist nach heutiger Erkenntnis ein Zustand, bei dem es auf Grundlage einer genetischen Prädisposition (drei Genloci sind inzwischen identifiziert und bis hin zu den relevanten Punktmutationen genau charakterisiert, weitere werden sicherlich demnächst identifiziert werden) $)^{3} \mathrm{zu}$ Migräneattacken kommt, die in ihrer Dynamik biochemisch ebenfalls inzwischen gut charakterisiert sind und die mit Schmerzen, mit vegetativen Symptomen und gelegentlich auch mit neurologischen Ausfallsymptomen (sog. Auren)

3 Estevez/Gardner (2004). 
einhergehen. ${ }^{4}$ Der Zustand der Attacke führt zu einer erheblichen Beeinträchtigung der Lebensqualität und manchmal sogar zu einem Ausfall der Fähigkeit, sich um persönliche Angelegenheiten zu kümmern, somit zu einem Ausfall der sozialen und produktiven (incl. reproduktiven) Funktionen. ${ }^{5}$

Diese genetische Prädisposition weisen über $20 \%$ der Bevölkerung auf, wobei bei ca. 15\% der erwachsenen Frauen und 5\% der erwachsenen Männer dadurch regelmäßig Migräneattacken auftreten (durchschnittlich einmal im Monat mit durchschnittlich einem Tag Dauer). ${ }^{6}$ Dieser (rein epidemiologische) Geschlechtsunterschied lässt sich auf den unterschiedlichen Einfluss von Triggerfaktoren für die einzelnen Attacken zurückführen. Allein schon die Prävalenz der Prädisposition lässt anfragen, ob ein so häufig verbreiteter Zustand als Krankheit aufgefasst werden darf. Zusammenfassend kann der Zustand einer Migräneattacke sicherlich als ein „Selektions“nachteil aufgefasst werden.

Nun kann man auf mindestens zwei Ebenen anfragen, ob diese genetische Prädisposition nicht gleichzeitig auch einen „Selektions“vorteil darstellt, zumindest angesichts der menschlichen Evolution. Eine Ebene betrifft die Migräne als individuelle Erkrankung. Es konnte gezeigt werden, dass Menschen mit einer Veranlagung für Migräne zwischen den Attacken eine fehlende Habituation bei der Verarbeitung kognitiver Reize aufweisen. ${ }^{7}$ Dies bedeutet, dass bei repetitiver Reizpräsentation im Verlauf der Kategorisierung eines Reizes die Reaktion auf einen Reiz bei Menschen mit Migräne um mehrere Millisekunden schneller erfolgt als bei Menschen ohne Migräne. Dies lässt sich leicht als „Selektions“vorteil, z. B. in Gefahrensituationen, vorstellen. Auf den (naheliegenden) Einwand, dass nicht die Prädisposition der Migräne an sich, sondern nur der Zustand der Attacke als „krank“ zu bezeichnen sei, lässt sich inhärent entgegnen, dass die Attacke aus physiologischer Sicht (was hier aus Platzgründen nicht weiter ausgeführt werden kann) als notwendige Unterbrechung interpretiert werden kann, um diese besonderen kognitiven Fähigkeiten zwischen den Attacken aufrechterhalten zu können. ${ }^{8}$

Auf einer zweiten Ebene lässt sich Migräne aufgrund sozialreproduktiver Aspekte als „Selektions“vorteil beschreiben. Ca. 80\% der Frauen mit Migräne haben während einer Schwangerschaft keine Migräneattacken und fühlen sich auch in weiteren Dimensionen subjektiv besser (z. B. leistungsfähiger, affektiv weniger belastet). ${ }^{9}$ Es gibt somit Frauen, die den Zustand einer Schwangerschaft geradezu anstreben, um für eine Zeit keine Migräne zu haben und sich subjektiv wohler zu fühlen. Dies ist heutzutage sicherlich eher die Ausnahme unter allen Frauen mit Migräne (in der Realität einer Kopfschmerzambulanz aber immer noch anzutreffen), mag aber unter anderen Lebensbedingungen eine evolutionäre Rolle gespielt haben.

\footnotetext{
Goadsby/Lipton/Ferrari (2002).

Leonardi et al. (2005).

Stovner et al. (2006).

Gantenbein/Sandor (2006).

Evers et al. (1999).

Silberstein (2004).
} 
Die grundsätzliche Frage, warum chronische Erkrankungen mit einer hochprävalenten genetischen Prädisposition bzw. Zustände mit genetischen Polymorphismen nicht aussterben, muss immer auch Anlass sein zu überprüfen, ob durch diese Prädisposition nicht auch ein „Selektions“vorteil besteht und somit die Prädisposition an sich nicht als (allein) krankhaft bezeichnet werden darf. Inzwischen beschäftigt sich eine ganze (nicht nur neurologische) Forschungsrichtung mit dieser Frage und bestätigt diesen ambivalenten Charakter von chronischen genetischen „Erkrankungen“. Dies sei auch noch an einem weiteren Beispiel, nämlich dem der Hyperhomocysteinämie, erläutert.

Die Hyperhomocysteinämie ist ein Zustand, bei dem im Blut ein erhöhter Spiegel der Aminosäure Homocystein besteht. Dieser Zustand ist verbunden mit einem erhöhten Risiko für Arteriosklerose und damit sekundär mit vaskulären Erkrankungen wie Herzinfarkt oder Schlaganfall. ${ }^{10}$ Da der Homocysteinspiegel direkt auch von den Vitaminen B12 und Folsäure beeinflusst werden kann, gab es für einige Jahre ein hohes kommerzielles Interesse, den Zustand der Hyperhomocysteinämie an sich als Krankheit zu klassifizieren und als Therapie die hochdosierte Gabe eben dieser Vitamine zu empfehlen. Hintergrund der Hyperhomocysteinämie ist in vielen Fällen eine genetische Variante (sog. C677T-Polymorphismus) im MTHFR-Gen. Dieses Gen kodiert für ein Enzym, das den Abbau von Homocystein aus dem Blut reguliert. Von den drei verschiedenen Polymorphismus-Varianten im MTHFR-Gen (CC, TT, CT) ist der TT-Polymorphismus am stärksten mit Arteriosklerose assoziiert. Die Prävalenz des TT-Polymorphismus ist allerdings in erster Linie ethnisch bedingt. So liegt sie im mediterranen Gebiet bei $18 \%$, in Nordeuropa bei $7 \%$, in Asien bei $4 \%$ und in Afroamerika bei $\%$. Es stellt sich nun die Frage, ob eine vermehrte Arteriosklerose, eine Hyperhomocysteinämie, der TT-Polymorphismus oder gar die ethnische Zugehörigkeit selbst eine Krankheit darstellt. Man wird sich schnell darauf einigen können, dass eine ethnische Zugehörigkeit als Krankheit mit unserem Verständnis des Krankheitsbegriffs nicht vereinbar ist. Der Polymorphismus selbst kann auch nicht nur als „Selektions“nachteil angesehen werden, da bekannt ist, dass die TT-Variante mit einer verbesserten Energiebereitstellung in Hungerphasen verbunden ist, also dann einen Vorteil darstellt, wenn längere Zeit auf eine Zufuhr von Kalorien verzichtet werden muss. In Form einer Medienkampagne ist durch verschiedene Interessenten die Hyperhomocysteinämie als Krankheit definiert und präsentiert worden. Interessanterweise haben aber die Interventionsstudien, die zu einer Senkung des Homocysteins durch die Gabe von Vitaminen im Blut geführt haben, nicht gleichzeitig auch zu einem Rückgang der Arteriosklerose, zu einer Senkung von vaskulären Erkrankungen oder zu einem sonst irgendwie messbaren Vorteil für die Betroffenen geführt. Hier fehlt ein wesentliches Merkmal von Krankheit, dass nämlich die (und wenn nur theoretisch vorstellbare) Reversibilität eines krankhaften Zustands mit einer Verbesserung der Krankheitsfolgen einhergehen muss.

10 Herrmann/Herrmann/Obeid (2007). 


\section{Neurodegeneration: Altern oder Krankheit?}

Der Münchener Demenzforscher Hans Förstl hat noch jüngst in der Wochenzeitung Die Zeit (39/2006) formuliert: „Die Wahrscheinlichkeit, bis zum Alter von 100 Jahren eine Demenz zu entwickeln, beträgt fast $100 \%$. Es stellt sich also die Frage, ob es sich nicht um den natürlichen Alterungsprozess des Menschen handelt."

Es ist inzwischen auch vielfältig in den Laienmedien verbreitet worden, dass alle Menschen irgendwann einmal an Morbus Alzheimer erkranken würden, wenn sie nur alt genug würden. Diese gleichzeitig trivial und unwissenschaftlich wirkende Aussage entspricht im Kern jedoch dem heutigen Erkenntnisstand über neurodegenerative Erkrankungen im Allgemeinen und lässt somit auch hier die Frage zu, ob ein Zustand, der häufig vorkommt und einer physiologischen Dynamik unterliegt, als Krankheit bezeichnet werden kann.

Die Prävalenz des Morbus Alzheimer steigt exponentiell mit zunehmendem Lebensalter ${ }^{11}$ und steht allein schon aufgrund der demographischen Entwicklung in Deutschland im zunehmenden Fokus der neurobiologischen Forschung. Der dem Morbus Alzheimer zugrundeliegende pathophysiologische Mechanismus ist nicht vollständig aufgeklärt, es handelt sich jedoch unter anderem um Amyloidablagerungen, die bei jedem Menschen mit zunehmendem Alter vorkommen und mit dem Ausmaß der kognitiven Beeinträchtigungen korrelieren. ${ }^{12}$ Interessanterweise kommen identische oder ähnliche Ablagerungen auch bei anderen neurodegenerativen Erkrankungen (wie z. B. der Lewy-Body-Demenz oder der frontotemporalen Demenz) vor, die sich in den sehr späten Stadien weder klinisch noch histopathologisch voneinander differenzieren lassen. ${ }^{13}$ Insofern ist die Diagnose eines Morbus Alzheimer im Spätstadium ein Konstrukt aufgrund des Verlaufs der Erkrankung und nicht eine eindeutige Beschreibung eines pathologischen Zustands. Ähnliche Beschreibungen sind z. B. auch für den Morbus Parkinson möglich, bei dem die Degeneration von Neuronen der Substantia nigra direkt mit dem Ausmaß der motorischen Beeinträchtigung durch diese Erkrankung korreliert, ${ }^{14}$ eine solche Degeneration aber bei jedem Menschen schon ab dem 20. Lebensjahr stattfindet und nur unterschiedlich rasch in ihrer Progression ist. Für die Definition des Krankheitsbegriffs ergeben sich nunmehr zwei Problemfelder. Wo ist die Grenze zwischen normaler altersbedingter kognitiver Beeinträchtigung und pathologischer Beeinträchtigung durch Morbus Alzheimer? Ist die Tatsache einer abnormen, frühzeitigen und rasch progredienten kognitiven Beeinträchtigung (i. e., die Diagnose eines Morbus Alzheimer) ein Krankheitszustand oder nur Ausdruck eines raschen und vorzeitigen Alternsprozesses, der in späterer und verzögerter Form gesellschaftlich nicht als Krankheit per se interpretiert würde?

\footnotetext{
11 Van der Flier/Scheltens (2005).

12 Blennow/de Leon/Zetterberg (2006).

13 Rachakonda/Pan/Le (2004).

14 Smits/Burbach/Smidt (2006).
} 
Der aktuelle Antwortversuch der Neurologie auf diese Fragen ist mehrgestaltig. Zum einen werden kognitive Veränderungen sowie biologische Marker (z. B. Demenzmarker im Liquor sowie morphologische Veränderungen im Gehirn, nachgewiesen durch MRT oder funktionell durch PET) quantifiziert und zum Altersdurchschnitt in Beziehung gesetzt. ${ }^{15}$ Somit ergibt sich eine statistische Definition des Unterschieds zwischen Krankheit und normalem Alternsprozess. Auf die Problematik dieses Ansatzes wird in Kapitel 6 noch einzugehen sein. Zum anderen werden genetische Prädispositionen als Voraussetzung für einen frühzeitigen und raschen kognitiven Abbau als eigentliche Krankheitsursache definiert. Hierzu gehören z. B. die Polymorphismen des ApoE-Lipoproteins und die sehr seltenen autosomal-dominant vererbten Formen des familiären $M$. Alzheimer. ${ }^{16}$ Es lässt sich also aus neuromedizinischer Sicht nicht exakt konstruieren, welcher Zustand bei der Neurodegeneration als „krankhaft“ oder als „physiologisch“ zu bezeichnen wäre.

\section{Krankheit: Definition oder pathophysiologisches Konstrukt?}

Bei der engen Verzahnung der Begriffe „Krankheit“ und „Diagnose“ stößt man häufig an die Grenze der Unterscheidbarkeit und muss feststellen, dass sich eine Krankheit nur über den Begriff einer Diagnose beschreiben lässt und dass es eben diese Diagnose ist, die die Krankheit konstituiert. Somit stellt sich die Frage, wie man formal eine Diagnose stellt (wenn dies eben nicht über klare Krankheitsprozesse geschieht), und damit die Frage, inwieweit Krankheiten auch definierte Konstrukte und nicht psychologische oder biologische Phänomene sind. Eine physiologische Determinierung von biologischen Erkrankungen ist also (zumindest nach dem heutigen Stand der Medizin) nicht immer möglich.

Dies berührt die Frage von diagnostischen Kriterien und damit die Frage, wie stark eine Krankheit durch die jeweils gültigen diagnostischen Kriterien oder eben durch biologische (im medizinischen Sprachgebrauch objektivierbare und messbare) Prozesse beschrieben wird. Dies soll ebenfalls am Beispiel der Krankheit Migräne dargestellt werden. An diesem Beispiel kann zusätzlich auch erläutert werden, inwieweit sich Krankheitsdefinition und medizinische Forschung im historischen Kontext direkt wechselseitig beeinflussen. Für die Migräne hat sich herausgestellt, dass eine ätiologische Klassifikation untauglich ist, da verwertbare biologische Marker bislang fehlen.

Die Migräne ist kein neuer Gegenstand der Medizingeschichte. Es gibt bereits zahlreiche Werke, die sich mit ihrer Phänomenologie beschäftigen, dies um so eindrucksvoller, als die Migräne seit der Frühgeschichte der Menschheit in verschiedenen Kulturen beschrieben wird und Anlass zu teilweise kuriosen Darstellungen gegeben hat, die auch Eingang in schöngeistige Literatur, Kunst und sogar Musik gefunden haben. ${ }^{17}$ Allerdings ist trotz der Vorstellung, dass es

15 Hsu et al. (2001), Wiltfang et al. (2005).

16 Poirier (2005), Williamson/LaRusse (2004).

17 Friedman (1972), Patterson/Silberstein (1993), Pöldinger/Kocher (1993). 
eine idiopathische Kopfschmerzentität wie die Migräne geben muss, das biologische Konstrukt bis etwa Anfang der 1990er Jahre ungeklärt geblieben.

Die ersten systematischen Beobachtungen über die Migräne haben Hippokrates $^{18}$, Celsus und Aretaeus gegeben. Interessanterweise äußern sich alle nicht explizit zur Pathogenese. ${ }^{19}$ Aretaeus spricht als erster von „heterocrania“, die er als eine Form der Cephalalgia im Gegensatz zur chronischen Cephalea interpretiert. Galen schließlich verwendet in seiner Decompositione Medicamentorum Secundum locos um 180 n. Chr. als erster den Begriff „, hemicrania“, von dem sich über die französische Prosodie unser heutiges Wort Migräne ableitet. Er diskutiert auch als Erster vage pathogenetische Vorstellungen, indem er Migräne auf ein Aufsteigen von zu vielen zu heißen oder zu kalten Dünsten zurückführt. ${ }^{20}$ Diese der Galenschen Variante der Humoralpathologie verpflichtete Ansicht wird von Caelius Aurelianus tradiert und findet sich in vielen Traktaten der arabischen Medizin und der Klostermedizin. Allen ist gemeinsam, dass als Ursache der Migräne ein Überangebot an Säften für den Kopf - vor allem an gelber Galle - angenommen wird. Oliver Sacks (1995) unterscheidet dabei eine rein humoralpathologische von einer sympathetisch-neurogenen Theorie, bei der der Ursprung der Migräne in peripheren Organen liegt, von wo aus sie sich durch Flüssigkeitswanderung über die Nerven im Kopf festsetzt. Die Migräne, die von allen antiken Autoren phänomenologisch sehr ähnlich beschrieben wird, wird hier also als eine Krankheit nach humoralpathologischen Vorstellungen beschrieben, ohne dass eine definitorische Festlegung erfolgt, wie eigentlich die Diagnose Migräne zu stellen ist.

Jason Pratensis (1486-1558) hat mit seinem Buch De cerebri morbis (1549) die Grundlage der Neurologie als eigenständiger Disziplin gelegt. ${ }^{21}$ Er beschreibt ausführlich die Migräne, bleibt aber bei der humoralpathologischen Theorie eines Überangebots an schwarzer Galle und Schleim. Bemerkenswert ist, dass er als Erster die Meningen als morphologisches Substrat der Kopfschmerzen identifiziert. Dabei wird die Migräne als eigenständiges Krankheitsbild wie bei den antiken Autoren beschrieben, das heißt, es gibt die gemeinsame Annahme einer Krankheit Migräne, nur die Definition und damit die Diagnosestellung ändern sich. Pratensis gibt auch eine Klassifikation von Kopfschmerzen mit diagnostischen Kriterien und akzeptiert erstmals explizit die Migräne als eine definierte Erkrankung.

Samuel Tissot (1728-1797) beschreibt 1780 im Traité des nerfs et de leurs maladies am ausführlichsten die Migräne und führt sie auf Irritationen des Magens zurück, die nerval über supraorbitale Nerven ins Gehirn ausstrahlen, wo sie einen Migräneanfall triggern können. Er unterscheidet dabei eine echte Migräne („migraine vraie“) von einer sekundären Migräne („migraine accidentelle“), die Symptom einer okulären oder rhinologischen Erkrankung ist. ${ }^{22}$ Damit wird die Krankheit neu definiert und andere (wahrscheinlich sympto- 
matische Kopfschmerzen) werden jetzt per definitionem auch als Krankheit Migräne akzeptiert.

Die Abhandlungen über die Migräne im 19. Jahrhundert diskutieren vor allem die Pathophysiologie der Migräne, ohne sich auf eine einheitliche Definition zu verständigen. Beispielhaft seien hier nur genannt: Peter Latham (1789-1875), der um 1850 Migräne als Folge einer arteriellen Konstriktion im Bereich der Arteria cerebri posterior ansieht; Hughlings Jackson (1834-1911), der Migräne um 1860 als Abart der Epilepsie beschreibt; Emil du Bois-Reymond (1818-1896), der 186o Migräne als Tetanie der Gefäßmuskulatur auffasst und damit die Zwei-Phasen-Theorie nach Harold Wolff mit initialer Vasokonstriktion und sekundärer Vasodilatation vorbereitet. Schließlich muss auch der Beitrag von William Gowers erwähnt werden, der 1886 in Diseases of the Nervous System keinen Beweis für eine vaskuläre Ursache findet. Er lokalisiert die gestörte neuronale Aktivität im Cortex (nicht wie Edward Liveing in den Thalamus), nimmt aber auch eine Ausbreitung in subkortikale Zentren an. Das für die Pathogenese-Diskussion des 19. Jahrhunderts wohl bedeutendste Werk ist jenes von Edward Liveing On Megrim, Sick-Headache, and Some Allied Disorders (1873); jedoch gibt auch er keine operationalisierbare Definition der Migräne, sondern geht implizit von einer Konvention über die Krankheit Migräne aus.

Erst in den 1940er und 1950er Jahren ist der erneute Versuch gemacht worden, alle Kopfschmerzformen systematisch zu klassifizieren. So haben Harold Georg Wolff (1948) im englischsprachigen Raum und Hartwig Heyck (1958) im deutschsprachigen Raum in ihren Monographien Einteilungen von Kopfschmerzen vorgeschlagen, die aber noch unsystematisch waren und sich nicht durchsetzen konnten. Auf Grundlage des Vorschlags von Wolff erstellte dann ein Ad Hoc Committee des National Institute of Neurological Diseases and Blindness (1962) in den USA eine Klassifikation, die in ihrer Struktur ätiologisch aufgebaut war. Danach wurden Kopfschmerzen, die vaskulär bedingt sind, unterschieden von Kopfschmerzen, die durch Muskelverspannung oder durch andere Faktoren bedingt sind; insgesamt wurden 15 Gruppen von Kopf- und Gesichtsschmerzen definiert. Diese Klassifikation hat für viele Jahre Bezeichnungen etabliert, die auch noch in heutigen neurologischen Lehrbüchern zu finden sind, so etwa die komplizierte Migräne, die gemeine Migräne, die klassische Migräne, aber auch den Muskelkontraktionskopfschmerz.

Das Problem der Klassifikation des Ad Hoc Committee war, dass sie sich auf spekulative Annahmen zur Pathophysiologie stützte und diese als systematisierendes Kriterium verwendete. So wurde die Migräne als primär vaskulär angesehen, nur weil sie einen pulsierenden Charakter haben kann. Eine weitere Schwäche dieser Klassifikation war, dass sie keine operationalisierten Kriterien verwendete, um die einzelnen Kopfschmerzformen zu definieren. Sie ist auch nicht verbindlich in andere Sprachen übersetzt worden. So hat sie nicht zu einer einheitlichen Forschungsaktivität beitragen können; die individuellen Definitionen der Migräne in den Publikationen der 196oer und 1970er Jahre wichen daher weiterhin relevant voneinander $\mathrm{ab}$.

Im Jahr 1988 wurde erstmals von der International Headache Society eine Klassifikation vorgelegt, die die verschiedenen Kopfschmerzformen nach phänomenologischen Gesichtspunkten klassifiziert und zudem einheitliche opera- 
tionalisierte Kriterien beinhaltet, nach denen die Kopfschmerzen exakt diagnostiziert werden können (Headache Classification Committee 1988). Die Kriterien sind so angelegt, dass eine hohe Spezifität unter Inkaufnahme einer geringeren Sensitivität erzielt wird (d.h. die Diagnose soll sicher stimmen, auch wenn nicht alle Betroffenen von den Kriterien erfasst werden). Klassifiziert werden sollen die Kopfschmerzen an sich und nicht die Patienten, die an den Kopfschmerzen leiden, daher wird ausdrücklich auch das gleichzeitige Vorliegen von mehreren Kopfschmerzdiagnosen akzeptiert. Dadurch haben zahlreiche Patienten, die vorher unter der Diagnose Migräne klassifiziert werden konnten, ihre Diagnose verloren. Dabei war noch ungeklärt, ob diesen Kopfschmerzen auch ein anderer biologischer Prozess zugrunde liegt.

Die Klassifikation von 1988 teilte Kopfschmerzen erstmals in primäre (= idiopathische) und in sekundäre (= symptomatische) Formen ein; insgesamt wurden 13 Gruppen unterschieden mit insgesamt über 160 verschiedenen Kopfschmerzdiagnosen. Gleichzeitig wurden erstmals auch Hinweise gegeben, wo noch keine ausreichende Validierung von bestimmten Kopfschmerzformen erfolgt war oder wo das Konzept einer bestimmten Entität (wie z. B. dem Kombinationskopfschmerz) sowohl aus phänomenologischer als auch aus ätiologischer Sicht in Frage gestellt werden musste.

Die exakten Definitionen der IHS-Klassifikation haben in den folgenden Jahren eine enorme Forschungstätigkeit beflügelt und z.T. überhaupt erst möglich gemacht. Durch einheitliche Kriterien konnten sowohl valide epidemiologische Studien als auch große klinische Studien durchgeführt werden. Die Entwicklung der modernen Pharmakotherapie der Migräne wäre beispielsweise ohne die Klassifikation nicht denkbar. Insgesamt hat sich gezeigt, dass die definitorische Klassifikation der Krankheit Migräne zu einer biologischen Forschung geführt hat, die weitgehend bestätigt hat, dass die Definition der Migräne auch einer biologischen Entität entspricht.

Im Jahr 2003 wurde eine Revision der alten IHS-Klassifikation von 1988 verabschiedet (Headache Classification Committee 2003). Diese Revision war aus mehreren Gründen notwendig geworden. So wurden bei einigen idiopathischen Kopfschmerzformen neue Erkenntnisse gewonnen, die zu einer Abänderung von diagnostischen Kriterien führen mussten. Weiterhin sind in der Zwischenzeit neue Entitäten identifiziert und wissenschaftlich kategorisiert worden. Außerdem sollten zu kontroversen Fragen, so etwa zur Existenz einer sog. Chronischen Migräne, Kriterien entwickelt werden, um epidemiologische und pathophysiologische Forschung zu initiieren, die diese Kontroversen klären soll.

Die neue IHS-Klassifikation teilt die Kopfschmerzen in insgesamt 14 Gruppen ein. Unverändert wird zwischen primären und sekundären Kopfschmerzen unterschieden. Die Grundkriterien der Migräne mussten dabei nicht verändert werden, sie haben sich wie gesagt als sehr robust herausgestellt. Lediglich das Kriterium „,verstärkt sich bei körperlicher Aktivität“ ist ergänzt worden durch „oder führt zu deren Verhinderung“. Dadurch kann nunmehr bei einigen sehr wenigen Menschen, die in der Klassifikation von 1988 noch nicht als Migräne klassifiziert worden sind, die Diagnose Migräne gestellt werden. Mit anderen Worten: Diese Patienten haben nun eine Krankheit, 
die sie vorher per definitionem nicht hatten. Als wichtigste neue Subform der Migräne ist dabei die sogenannte Chronische Migräne eingefügt worden, die vorher ausdrücklich nicht zulässig war. So ist eine neue Krankheitsform durch eine Definition geschaffen worden, weil es sich gezeigt hatte, dass das Phänomen existiert, aber nicht in der (mit einem allumfassenden Anspruch auftretenden) Klassifikation enthalten war.

Zusammenfassend kann also festgestellt werden, dass die Zuordnung der Krankheit Migräne zu einem betroffenen Patienten historisch von der jeweils gültigen Definition abhing und insofern beliebig war. ${ }^{23}$ Zuordnungen desselben Zustands zur Diagnose Migräne waren insofern im Laufe der Medizingeschichte häufig Schwankungen unterworfen. Die stufenweise Weiterentwicklung der Migränedefinition (jeweils sowohl Voraussetzung als auch Ergebnis von biologischer Forschung) hat inzwischen zu einer weitgehenden Übereinstimmung der Diagnosestellung anhand semiologischer Kriterien mit den heute bekannten biologischen Mechanismen geführt. Allgemein gesprochen können also streng phänomenologische und streng ätiologische Krankheitsklassifikationen biologische Zustände häufig nicht adäquat abbilden, phänomenologische Krankheitsbeschreibungen und ätiologische Forschung können sich aber gegenseitig beeinflussen.

\section{Statistische Methoden zur Normierung von Krankheit}

Krankheitsbilder, die sich mit quantifizierbaren Messmethoden erfassen lassen, unterliegen in ihrer Normierung auch einer Willkürlichkeit von statistischen Definitionen. In den Neurowissenschaften berührt dies z. B. neuropsychologische Funktionen, die mit psychometrischen Messverfahren erfasst werden. Pathologische Ergebnisse dieser Messverfahren werden anhand von sogenannten Normwerten definiert. Diese Normwerte sind allerdings ein Konstrukt, das sich aus Berechnungen von Ergebnissen einer gesunden Kontrollgruppe ergibt. Hierbei kann eo ipso nicht ermittelt werden, ob diese Kontrollgruppe auch wirklich gesund ist, da die Abgrenzung von gesund zu krank erst nach ihrer Untersuchung erfolgen kann. So ist es in der Medizin üblich geworden, bei parametrisch verteilten Messwerten die Normgrenze beim arithmetischen Mittelwert \pm zwei Standardabweichungen festzusetzen. Diese bedeutet aber auch, dass ca. $5 \%$ der gesunden Normalbevölkerung per definitionem pathologische Messwerte aufweist. Nun kann man entgegnen, dass pathologische Messwerte natürlich keine Krankheit konstituieren. Dies entspricht aber nicht immer dem medizinischen Alltag, der oftmals auf eine pragmatische Diagnosebegründung angewiesen ist (die im Übrigen auch von Patienten und dem Gesundheitssystem oftmals eingefordert wird).

Als weiteres Problem kommt bei der statistischen Normierung von Krankheitszuständen hinzu, dass häufig nicht nur eine quantifizierbare Untersuchung, sondern deren mehrere durchgeführt werden. Je mehr solcher Erhebungen jedoch vorgenommen werden, desto größer ist die Wahrscheinlichkeit, dass ein pathologisches Messergebnis bei einem an sich gesunden Menschen erhoben wird. Wie oben gesagt, liegt die Wahrscheinlichkeit für ein

23 Evers (2004). 
pathologisches Ergebnis bei einem gesunden Menschen bei ca. $5 \%$, wenn ein Test durchgeführt wird. Werden jetzt 1o Tests durchgeführt (was in der Labormedizin durchaus üblich ist), so erhöht sich die Wahrscheinlichkeit für wenigstens ein pathologisches Ergebnis bereits auf ca. $20 \%$. Teilweise wird diesem Problem begegnet, indem für Untergruppen eigenständige Normwerte festgelegt werden (so nach Geschlecht, nach Alter, nach Ethnie). Eine solche Differenzierung stößt aber notwendigerweise irgendwann an ihre Grenzen.

Weiterhin unterliegt die statistische Normierung auch genetischen, ethnischen und soziokulturellen Einflüssen. Diese Zusammenhänge sollen an einigen Beispielen noch einmal skizziert werden. So ist auf die Hyperhomocysteinämie bereits weiter oben eingegangen worden. Der Normwert des Homocysteinspiegels im Blut ist somit abhängig vom genetischen Polymorphismus (oder sollte für jeden Polymorphismus ein eigenständiger Normwert festgelegt werden?); außerdem ist er abhängig von Ernährungsverhalten und der Zuführung von bestimmten Vitaminen (ohne dass diese einen Einfluss auf den durch die Hyperhomocysteinämie vermittelten krankhaften Zustand haben). Ein anderes Beispiel ist die Hypercholesterinämie, die stark von ethnischen Einflüssen abhängt (so ist der Cholesterinspiegel in ostasiatischen Völkern sehr viel niedriger). Gleichzeitig ist der Grenzwert der Hypercholesterinämie auf $200 \mathrm{mg} / \mathrm{dl}$ festgelegt worden. Dies ist zum einen gut zu merken, zum anderen ist er niedriger, als es die gängige Regel vom arithmetischen Mittelwert \pm zwei Standardabweichungen erfordern würde. Es soll an dieser Stelle auch auf die Debatte verwiesen werden, inwieweit Interessen der pharmazeutischen Industrie zur Festlegung dieses niedrigen Grenzwerts mit beigetragen haben.

Somit ergeben sich einige Anfragen an die Definition der statistischen Norm als Grundlage für eine Krankheitsdefinition. Wie kann z. B. eine statistische biologische Norm definiert werden, wenn sie altersabhängig ist? Dies berührt die Frage, was einen gesunden alten Mensch charakterisiert. Unabhängig von dem Verfahren der arithmetischen Berechnung eines Normwerts muss überlegt werden, vor welchem Hintergrund die Grunddaten für eine solche Berechnung erhoben werden. Weiterhin muss gefragt werden, wie sich statistische und biologische Norm zueinander verhalten. Notwendigerweise gibt es per definitionem statistisch kranke Menschen, die gesund sein müssen (wenn man den oben beschriebenen arithmetischen Normwert heranzieht). Darüber hinaus muss aber auch bedacht werden, dass sich biologisch krankhafte Zustände einer messbaren Normabweichung entziehen können. Abschließend soll auch darauf hingewiesen werden, dass trotz aller berechtigten Kritik und nur relativen Bedeutung des statistischen Krankheitsbegriffs dieser auch Vorteile aufweist. So ist er gut operationalisierbar und somit $z$. B. für eine statistische Prognoseabschätzung geeignet. Zudem ist er auch kategorisierbar und damit z. B. für epidemiologische Forschung geeignet.

\section{Symptom: pathologisch oder notwendig?}

Es soll noch auf ein Phänomen hingewiesen werden, das ebenfalls zur Relativität von Krankheit beiträgt. So gibt es aus Sicht der Neurowissenschaften Zustände, die als Symptom zwar landläufig als pathologisch bzw. krankhaft klassifiziert werden, im biologischen System des Menschen aber (überlebens-) 
notwendig sind und daher nur schwer einer Krankheit zugeordnet werden können.

Am besten ist dies am Beispiel des Schmerzes als Symptom zu erläutern. Man kann sich wahrscheinlich schnell darauf verständigen, dass leichter, vorübergehender Schmerz als Warnsignal eher physiologisch und nicht als krankhafter Zustand zu werten ist und dass eine Chronifizierung von Schmerzen bzw. eine durch traumatische Zufügung übermäßige Intensität von Schmerzen als Krankheitszustand zu interpretieren ist. Im Übergang kann es manchmal sehr schwer zu differenzieren sein, wann ein Schmerz physiologisches Warnsymptom ist und wann er einen eigenständigen Krankheitscharakter erfährt.

Es gibt den (außerordentlich seltenen) genetisch bedingten Zustand, dass ein Mensch keine C-Fasern aufweist, die für eine Weiterleitung chronischer Schmerzen zwingend notwendig sind. ${ }^{24}$ Diese Menschen verspüren zwar einen Akutschmerz, aber keinen (über A $\delta$-Fasern vermittelten) Dauerschmerz. Dieser Zustand ist mit dem Leben kaum vereinbar, da Schmerz als Warnsymptom bei Verletzung der körperlichen Integrität ausfällt, zumindest versterben diese Menschen sehr früh an Bagatelltraumen, Verbrennungen oder Ähnlichem. Auch hier stellt sich also wieder die Frage nach der Grenzziehung zwischen einem natürlichen physiologischen Zustand und einem krankhaften Zustand, die beide auf denselben (patho-)physiologischen Prozess zurückgeführt werden können. Ähnliche Beispiele ließen sich hier auch für andere Symptome finden, etwa der Juckreiz (mit einer sehr starken Analogie zum Symptom Schmerz), die Hautpigmentation (Ist Albinismus eine Krankheit?), das körperliche Erleben affektiver Symptome oder die Störung mnestischer Funktionen.

\section{Zusammenfassung}

Aus neurowissenschaftlicher Sicht ist eine strikte Grenzziehung zwischen Krankheit und Gesundheit nicht möglich. So wünschenswert eine operationalisierbare Definition von Krankheit auch auf vielen Ebenen unserer Gesellschaft und unseres Gesundheitssystems wäre, so ist es vielleicht doch klüger zu akzeptieren, dass es eine solche Definition im exakten Sinne nicht geben wird. Damit soll jedoch nicht einer Beliebigkeit des Zustands Krankheit das Wort geredet werden. In unserem reglementierten und starken sozioökonomischen Einflüssen ausgesetzten Gesundheitssystem ist es im Gegenteil vielmehr zwingend, von dieser Exaktheit abzusehen und Krankheit häufig als Kompromissformel zu definieren.

Die Wertigkeit eines biologischen oder psychischen Zustands (bzw. dynamischen Prozesses) als Krankheit hängt nicht nur von der objektiven Definition dieses Zustands und dem subjektiven Empfinden des Betroffenen ab, sondern auch von weiteren Faktoren, die nicht von einem Untersucher oder dem Betroffenen selbst beeinflussbar sind (z. B. situative Faktoren, soziale, historische und statistische Normen). Diese einzelnen Faktoren können zu einem

24 Sztriha et al. (2001). 
qualitativ unterschiedlichen Ergebnis bezüglich der Attribuierung desselben Zustands als Krankheit kommen. Krankheit ist eben nicht durch biologische Prozesse und das subjektive Empfinden des Menschen determiniert, sondern auch durch die Sichtweise eines objektiven Betrachters und durch die reziproken Reaktionen des Systems Mensch auf diese Prozesse (die je nach Lebensphase, Reproduktivitätsstatus etc. bei demselben Individuum unterschiedlich ausfallen können). Somit kann derselbe biologische Prozess, selbst bei derselben subjektiven Betrachtung durch den Betroffenen mal als „krank“ und mal als „gesund“ interpretiert werden.

\section{Literatur}

Ad Hoc Committee (1962): Ad Hoc Committee on Classification of Headache, Classification of headache, JAMA 179 (1962), p. $717 f$.

Blennow/de Leon/Zetterberg (2006): Kai Blennow, Mony J. de Leon, Henrik Zetterberg, Alzheimer's disease, Lancet 368 (2006), p. 387-403

Du Bois-Reymond (1860): Emil du Bois-Reymond, Zur Kenntnis der Hemikrania, Archiv für Anatomie, Physiologie und wissenschaftliche Medizin (1860), S. 461-468

Estevez/Gardner (2004): Miguel Estevez, Kathy L. Gardner, Update on the genetics of migraine, Human Genetics 114 (2004), p. 225-235

Evers et al. (1999): Stefan Evers, F. Quibeldey, Karl-Heinz Grotemeyer et al., Dynamic changes of cognitive habituation and serotonin metabolism during the migraine interval, Cephalalgia 19 (1999), p. 485-491

Evers (2004): Stefan Evers, Die neue IHS-Klassifikation, Hintergrund und Struktur, Schmerz 18 (2004), S. 351356

Friedman (1972): Arnold Friedman, The headache in history, literature and legend, Bulletin of the New York Academy of Medicine 48 (1972), p. 661-681

Gantenbein/Sandor (2006): Andreas R. Gantenbein, Peter S. Sandor, Physiological parameters as biomarkers of migraine, Headache 46 (2006), p. 1069-1074

Goadsby/Lipton/Ferrari (2002): Peter J. Goadsby, Richard B. Lipton, Michael D. Ferrari, Migraine - current understanding and treatment, New England Journal of Medicine 346 (2002), p. 257-270

Gowers (1886): William R. Gowers, A manual of diseases of the nervous system, Vol. 2, London 1886

Headache Classification Committee (1988): Headache Classification Committee, Classification and diagnostic criteria for headache disorders, cranial neuralgias and facial pain, Cephalalgia 8 (1988), p. 1-96

Headache Classification Subcommittee (2004): Headache Classification Subcommittee, The international classification of headache disorders, Cephalalgia 24 (2004), p. 1-160

Herrmann/Herrmann/Obeid (2007): Wolfgang Herrmann, Marcus Herrmann, Rima Obeid, Hyperhomocysteinaemia: a critical review of old and new aspects, Current Drug Metabolism 8 (2007), p. 17-31

Heyck (1958): Hartwig Heyck, Der Kopfschmerz. Differentialdiagnostik und Therapie für die Praxis, Stuttgart 1958

Hsu et al. (2001): Yuan-Yu Hsu, An-Tao Du, Norbert Schuff et al., Magnetic resonance imaging and magnetic resonance spectroscopy in dementias, Journal of Geriatric Psychiatry and Neurology 14 (2001), p. 145-166

Jilek-Aall (1999): Louise Jilek-Aall, Morbus sacer in Africa. Some religious aspects of epilepsy in traditional cultures, Epilepsia 40 (1999), p. 382-386

Karbowski (1986): Kazimierz Karbowski, Samuel Auguste Tissot. His research on migraine, Journal of Neurology 233 (1986), p. 123-125

Leonardi et al. (2005): Matilde Leonardi, Timothy J. Steiner, Ann I. Scher et al., The global burden of migraine: measuring disability in headache disorders with WHO's Classification of Functioning, Disability and Health (ICF), Headache Pain 6 (2005), p. 429-440

Liveing (1873): Edward Liveing, On megrim, sick-headache, and some allied disorders: A contribution to the pathology of nervestorms, London 1873 


\section{A Theoretische Grundlagen von Normalität und Krankheit}

Patterson/Silberstein (1993): Stephanie Patterson, Stephen D. Silberstein, Sometimes jello helps: Perceptions of headache etiology, triggers and treatments in literature, Headache 33 (1993), p. 76-81

Pearce (1986): John M. S. Pearce, Historical aspects of migraine, Journal of Neurology, Neurosurgery and Psychiatry 49 (1986), p. 1097-1103

Pestronk (1988): Alan Pestronk, The first neurology book, Archives of Neurology 45 (1988), p. 341-344

Pöldinger/Kocher (1993): Walter Pöldinger, Ralph Kocher, Schmerz in der Gesellschaft und in der Medizin, Schweizerische Rundschau für Medizinische Praxis 82 (1993), S. 255-259

Poirier (2005): Judes Poirier, Apolipoprotein E, cholesterol transport and synthesis in sporadic Alzheimer's disease, Neurobiology of Aging 26 (2005), p. 355-361

Pratensis (1549): Iason Pratensis, De Cerebri Morbis. Hoc Est, Omnibus Ferme, etc., Basel 1549

Rachakonda/Pan/Le (2004): Varun Rachakonda, Tian Hong Pan, Wei Dong Le, Biomarkers of neurodegenerative disorders. How good are they?, Cell Research 14 (2004), p. 347-358

Ritter (1969): Gerhard Ritter, Die Neurologie in der hippokratischen Medizin, Der Nervenarzt, 40 (1969), p. 327-333

Sacks (1985): Oliver Sacks, Migräne. Evolution eines häufigen Leidens, Stuttgart 1985

Silberstein (2004): Stephen D. Silberstein, Headaches in pregnancy, Neurologic Clinics 22 (2004), p. 727-756

Smits/Burbach/Smidt (2006): Simone Marije Smits, J. Peter Burbach, Marten P. Smidt, Developmental origin and fate of mesodiencephalic dopamine neurons, Progress in Neurobiology 78 (2006), p. 1-16

Stovner et al. (2006): Lars Jacob Stovner, John-Anker Zwart, Knut Hagen et al., Epidemiology of headache in Europe, European Journal of Neurology 13 (2006), p. 333-345

Sztriha et al. (2001): László Sztriha, Gilles G. Lestringant, Jozef Hertecant et al., Congenital insensitivity to pain with anhidrosis, Pediatric Neurology 25 (2001), p. 63-66

Tissot (1780): Samuel Auguste Tissot, Traité des nerfs et de leurs maladies. Tome III, Partie II, Paris 1780

Van der Flier/Scheltens (2005): Wiesje M. van der Flier, Philip Scheltens, Epidemiology and risk factors of dementia, Journal of Neurology, Neurosurgery and Psychiatry 76 (2005), p. v2-v7

Vanzan/Paladin (1992): Anna Vanzan, Francesco Paladin, Epilepsy and Persian culture. An overview, Epilepsia 33 (1992), p. 1057-1064

Williamson/LaRusse (2004): Jennifer Williamson, Susan LaRusse, Genetics and genetic counseling: Recommendations for Alzheimer's disease, frontotemporal dementia, and Creutzfeldt-jakob disease, Current Neurology and Neuroscience Reports 4 (2004), p. 351-357

Wiltfang et al. (2005): Jens Wiltfang, Piotr Lewczuk, Peter Riederer et al., Consensus paper of the WFSBP Task Force on Biological Markers of Dementia. The role of CSF and blood analysis in the early and differential diagnosis of dementia, The World Journal of Biological Psychiatry 6 (2005), p. 69-84

Wolff (1948): Harold George Wolff, Headache and other head pain, New York 1948 